\title{
Empleo de las estrategias de aprendizaje en la universidad. Un estudio de caso
}

\section{Use of Learning Strategies in the University. A Case Study}

\author{
Mitchell Alberto Alarcón Díaz*(i) \\ Universidad Nacional de Educación Enrique Guzmán y Valle, Lima, Perú \\ ORCID: https://orcid.org/0000-0003-0027-5701 \\ Noel Alcas Zapata \\ Universidad de San Martin de Porres, Lima, Perú \\ ORCID: https://orcid.org/0000-0002-2505-6778 \\ Henry Hugo Alarcón Diaz \\ Universidad Nacional de Educación Enrique Guzmán y Valle, Lima, Perú \\ ORCID: https://orcid.org/0000-0003-1588-4390 \\ José Arnin Natividad Arroyo \\ Universidad Nacional de Educación Enrique Guzmán y Valle, Lima, Perú \\ ORCID: https://orcid.org/0000-0002-1793-7495 \\ Antonio Rodríguez Fuentes \\ Universidad de Granada, Granada, España \\ ORCID: https://orcid.org/0000-0002-8036-9902
}

Recibido 20-09-18 Revisado 09-10-18 Aprobado 12-01-19 En línea 20-03-19

Correspondencia:

Email: malarcond@ucv.edu.pe

\section{Citar como:}

Alarcón, A., Alcas, N., Alarcón, H., Natividad J., \& Rodríguez, A. (2019). Empleo de las estrategias de aprendizaje en la universidad. Un estudio de caso. Propósitos y Representaciones, 7(1), 10-32. Doi: http://dx.doi.org/10.20511/pyr2019.v7n1.265

(C) Universidad San Ignacio de Loyola, Vicerrectorado de Investigación, 2019.

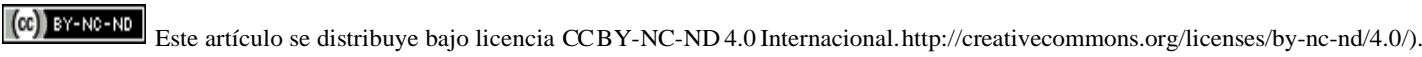




\section{Resumen}

El objetivo de este estudio fue caracterizar las estrategias de aprendizaje utilizadas por los estudiantes de educación superior de una universidad de Lima. Para lograr este propósito, el enfoque es cualitativo, el cual nos permite interpretar la praxis misma de los alumnos. La metodología de esta investigación incluyó la observación directa y la entrevista a profundidad, cuyo propósito fue comprender las razones de la puesta en práctica de determinadas estrategias. La conclusión de la investigación es que las estrategias metacognitivas, el procesamiento de la información y el control del contexto se convierten en las aristas fundamentales al momento de aprender por parte de los estudiantes.

Palabras clave: Estrategias; aprendizaje; estudio de caso; educación superior.

\section{Summary}

The objective of this study was the characterization of the learning strategies of higher education students of a university in Lima. To achieve this purpose, the focus is qualitative, which allows us to interpret the same practice of the students. The methodology of this research, including direct observation and in-depth interviewing, has the purpose of understanding the reasons for practice in the practice of strategies. The conclusion of the research is that the metacognitive strategies, the processing of information and the control of the context become the fundamental principles of the moment of learning by students.

Keywords: Strategies; Learning; Case Study; Higher Education.

\section{Introducción}

Las universidades peruanas están enfrentando el reto de desarrollar el enfoque del currículo por competencias, en donde paralelamente están en procesos institucionales de licenciamiento institucional y acreditación de cada una de sus escuelas de formación profesional. Sin embargo, en ese proceso de restructuración, también de manera sistemática, se está abordando la problemática de la deserción estudiantil y el bajo rendimiento académico de los estudiantes. Esto debido a que las Universidades, con sus docentes, no han incursionado todavía a fondo, para establecer estrategias didácticas que sean coherentes con los recursos y materiales de enseñanza, con los estilos de aprendizaje preferente, y la motivación intrínseca. En ese sentido, se necesita conocer cómo aprende el estudiante universitario, y qué otros recursos materiales y virtuales les son necesarios para la adquisición del conocimiento y la investigación. Se habla, entonces, de ciertas experiencias exitosas del contexto europeo, denominado "Espacio Europeo de Educación Superior (EEES)" (Rodríguez, 2008), en donde la investigación acción, ayuda a establecer y aplicar estrategias interinstitucionales, por ejemplo para la evaluación formativa de los estudiantes universitarios, empoderando al estudiante no solo en la construcción de sus aprendizajes propios sino también en su propia evaluación de los mismos (Gallego \& Rodríguez, 2017). Es decir, las universidades aplican estrategias en redes, para desarrollar sistemas e instrumentos de evaluación formativa en la docencia universitaria. Lo cual permite incorporar metodologías docentes que potencien en gran medida el aprendizaje autónomo de los estudiantes universitarios (Vallés, Ureña \& Ruiz, 2011). Esto implica potenciar las estrategias de aprendizaje. Pero para que ello ocurra, primero hay que conocerlas, identificar las dificultades, proporcionarle al estudiante todas 
las herramientas y recursos didácticos que se tienen disponibles y ayudarlo cuando sea necesario. Asimismo, el estudiante tiene que aprender a autorregular su aprendizaje, y socializarlo con sus pares, para luego aplicarlo en la solución de problemas de su entorno. En la base de ello está la competencia comunicativa del discente universitario de la que hay que tener constancia (Gallego \& Rodríguez, 2013, 2015; Rodríguez, Ayllón, Gallego \& Gómez, 2017) y la garantía de los espacios y recursos para materializar esta interacción entre ellos (Rodríguez, Rodríguez \& Gallego, 2015; Rodríguez \& Rodríguez, 2016).

Las estrategias de aprendizaje en el nivel universitario están centradas en el estudiante e implican centrar todo el proceso de cimentación del conocimiento, mucho más activo y autónomo, dada la madurez del aprendiz. En donde, el estudiante va regulando sus aprendizajes y seleccionando sus estrategias. Al respecto, Porto, Brenlla, Barca, Almeida, \& Peralbo (2012) sostienen que el aprendizaje autorregulado se orienta a procesos en los cuales el estudiante dirige metódicamente y controladamente sus operaciones, tendencias y emociones hacia el logro de sus metas planteadas. Schunk (1998) afirma que la autorregulación está muy ligada a variables motivacionales que influyen sobre ella, como son, por ejemplo, el establecimiento de metas, la autoeficacia, las expectativas que se generan, la actitud que se asume al orientarla a la meta, y el rendimiento académico posterior. Asimismo, una de las metas centrales de las universidades, en general, es que los estudiantes puedan controlar su proceso de aprendizaje y adquieran competencias que puedan utilizar dentro y fuera del entorno de la clase, y el eje central es el aprendizaje autónomo que le servirá para toda la vida (Fernández et al., 2013).

\section{La formación profesional universitaria.}

La universidad, como institución de formación de profesionales, tiene un rol que va más allá de la generación y profundización del conocimiento científico y la investigación. Su nuevo rol está enmarcado en funciones que implican un alto nivel de liderazgo para el desarrollo de otras maneras de educación. Ello requiere que se incorporen diferentes áreas del conocimiento científico: interdisciplinar y transdisciplinar, asociados al desarrollo sostenible, y dentro de un marco de preservación de los principios éticos. En esta línea de pensamiento, las universidades tienen como tarea, que le encomienda la sociedad en general, formar profesionales competentes, promover la investigación y la generar conciencia crítica que coadyuve a la difusión de conocimientos, actitudes y valores en el marco del desarrollo sostenible (UNESCO, 1998). Asimismo, se entiende que un adecuado modelo de desarrollo tiene que apoyarse en la ética y, fundamentalmente, en la solidaridad entre todos los seres humanos que pertenezcan a una comunidad, en particular, independientemente del tiempo y la generación a la que pertenezcan (Aznar Minguet, Ull, Piñero, \& Martínez-Agut, 2013).

Por otro lado, es necesario reconocer el rol que cumplen los estudiantes como agentes activos del sistema educativo universitario. Por esta razón, las universidades inciden en el desarrollo del currículo por competencias que incluyen habilidades y actitudes, en relación con el perfil profesional de la carrera. Todo ello con el propósito de cubrir las necesidades de la demanda social y la demanda del mercado laboral (Blázquez, Chamizo, Cano, \& Gutiérrez, 2013). También, en el proceso de formación profesional, se tiene en cuenta la evaluación de los estudiantes en el marco de los sistemas de calidad como una forma de aplicar ciertos estándares, que marquen las pautas para garantizar que los futuros profesionales adquieran las competencias necesarias para desempeñarse en el futuro, con eficiencia y eficacia. 
A las universidades, en la actualidad, se les presenta un nuevo reto: la responsabilidad social universitaria (RSU), en conexión con la empresa y con el ámbito público. En referencia con la empresa, tiene que establecer alianzas estratégicas para desarrollar conjuntamente proyectos de innovación tecnológica e investigación. En el ámbito público, se espera que atienda la demanda social al promover el desarrollo y practica de los valores éticos, el pensamiento crítico, la solidaridad y el desarrollo sostenible (Larrán \& Andrades, 2015).

La formación de los estudiantes universitarios se concreta a través de la educación formativa, la cual implica, a su vez, que se tenga en cuenta los perfiles de cada carrera, la visión y la misión de la universidad y en concordancia con las mallas curriculares que es inherente a cada profesión. Pero esto no es suficiente, los procesos de aprendizaje que, naturalmente, incluyen las estrategias de enseñanza y las estrategias de aprendizaje requieren que se enfoquen estos procesos para el desarrollo de cuatro pilares de la educación, como son: a) aprender a conocer, como un medio y finalidad humana, cuyo propósito es la adquisición de conocimientos para clasificarlos y codificarlos. Esto es, en esencia, lo que cada persona tiene que aprender a conocer del mundo que lo rodea y, al mismo tiempo, desarrollar sus capacidades, b) aprender a hacer, que implica, en el caso de los estudiantes universitarios, enseñarles a cómo poner en práctica lo aprendido y que se pueda adaptar fácilmente a su futuro mercado laboral, c) aprender a vivir con los demás, que en la práctica se ha convertido en uno de los principales propósitos de la educación actual, es decir, el profesional universitario tiene que desarrollar un conjunto de habilidades sociales para ser tolerante, solidario, asertivo, y que pueda transmitir y fomentar el conocimiento a los demás miembros de su comunidad, d) aprender a ser, lo cual acarrea que la educación debe ayudar al desarrollo global de la persona, para que le posibilite actuar de manera reflexiva y crítica, con pensamiento autónomo y responsabilidad individual (Delors, 1996).

\section{Estrategias de aprendizaje en el nivel universitario.}

Se entiende que las estrategias de aprendizaje son procedimientos, de carácter voluntario e intencional, que asume el estudiante para lograr un determinado objetivo. Muchas veces, el uso de estrategias de autorregulación del aprendizaje dependen, en gran medida, de la capacidad que percibe el estudiante, aunque también de otros factores personales como la motivación por aprender y, sobre todo, la percepción que se tiene acerca de la efectividad del uso de las estrategias de aprendizaje. En ese sentido, Bahamón, Vianchá, Alarcón \& Bohórquez (2012) sostienen que en el ambiente universitario las instituciones con sus docentes deben proporcionarles a sus estudiantes una proximidad a información del campo complejo, de manera que pueda llegar al procesamiento de la información y del conocimiento, de niveles mejor elaborados. Esto implica también, que utilice variadas estrategias con niveles de autonomía que garanticen de manera exitosa la culminación de sus estudios superiores. Las últimas reformas introducidas en la educación de nivel universitario, han permitido paralelamente, la utilización de estrategias y métodos como por ejemplo: el trabajo colaborativo como parte de la dinámica de equipos de estudio, las Tecnologías de la información, a través de recursos como el Internet y la Web 2.0 (Cabero \& Marín, 2013, García, Rodríguez \& Gallego, 2011).

En el nivel universitario, tratándose del desarrollo del enfoque curricular por competencias, se espera que los estudiantes utilicen ciertas estrategias de aprendizaje, que hasta cierto punto sean intencionadas, con el propósito de reforzar el aprendizaje autónomo y, por lo tanto, mejorar su rendimiento académico (García \& Tejedor, 2017). Estos procesos se dan de manera natural y pasan por la recuperación de los conocimientos previos y la recuperación de la 
información, donde se evidencian todo tipo de pensamientos, acciones, actitudes, motivaciones que sostienen este proceso.

Por otra parte, Herrera \& Lorenzo (2009) sostienen que en la educación universitaria el principal centro de atención es el estudiante, y, en ese contexto, las universidades deben atender los componentes del aprendizaje en el aspecto cognitivo, socioafectivo y motivacionales. Para ello tienen que proporcionarle todas las herramientas necesarias para que pueda adquirir el conocimiento y contribuir en la investigación científica como una de las actividades supremas de todo centro de formación superior universitario. En esta línea de pensamiento, Pintrich \& García (1993) afirman, en referencia a las estrategias de aprendizaje, que existen tres grandes tipos: a) Estrategias cognitivas que se orientan al repaso, la elaboración y organización de la información, y también el desarrollo del pensamiento crítico, b) Estrategias metacognitivas, en donde se planifica, revisa y ordenan las acciones utilizadas durante el proceso de aprendizaje, y c) las estrategias de regulación de recursos, que se refiere a la distribución del tiempo y del ambiente donde el estudiante estudia, ordena el esfuerzo, se establece el aprendizaje con pares y se puede solicitar ayuda si es necesaria.

\section{Sistema de evaluación formativa en la universidad.}

La evaluación es uno de los pilares de cualquier proceso de enseñanza - aprendizaje. En particular, en el sistema de educación superior universitaria, es relevante para que se puedan tomar decisiones sobre posibles cambios epistemológicos y hasta metodológicos, para dar respuestas concretas a las demandas sociales y las necesidades del mercado laboral. Se trata entonces de la formación de profesionales competentes que puedan aplicar con eficacia sus conocimientos adquiridos en la universidad. En ese sentido, se sustenta la nueva forma de percibir y aplicar la evaluación de los estudiantes en las universidades. Al respecto, Buscà, Pintor, Martínez, \& Peire (2010) sostienen que los nuevos estilos, que se están imponiendo en la docencia universitaria, se orientan fundamentalmente por la evaluación de carácter formativo y procesual o continua, es decir, que al margen de los conocimientos que adquiere el estudiante universitario, importa también la calidad de persona y los principios éticos que debe preservar y aplicar en su profesión y su mejora como tal profesional día a día.

Merece también la reflexión sobre la distinción entre evaluar y calificar. Evaluar es un proceso mediante el cual se recoge información para examinarla, emitir un juicio sobre ella y facilitar la posible toma de decisiones. Se da en todo el proceso de enseñanza - aprendizaje. En cambio, el proceso de calificar implica materializar un juicio emitido con una nota alfanumérica, que se le asigna a un sujeto. Este aspecto, es circunstancial y puntual, habitualmente se ejecuta al final. Por ello se dice que es sumativa (Hamodi, Pastor, \& Pastor, 2014), mientras que la evalaución deber ser continua, a guisa de proceso seguido y no solo de producto alcanzado. Por otra parte, uno de los problemas que se presenta con la aplicación de la evaluación formativa es, obviamente, la concepción que tienen algunos docentes universitarios sobre la evaluación, porque persisten las prácticas evaluativas tradicionales. Que son, a gran escala, finales, y que descuidan el desempeño del estudiante universitario por completo. La evaluación formativa, por lo tanto, tiene que acompañar al estudiantes mediante todo el proceso de aprendizaje (Rizo, 2013).

La eficacia del trabajo docente está asociada, de alguna manera, a la actitud y pericia de los maestros, tanto para evaluar los aprendizajes de sus estudiantes durante el trabajo en el aula, como para tomar decisiones que promuevan el aprendizaje y, sobre todo, que lo regulen en 
función a las necesidades de los estudiantes y de la demanda de la sociedad y la demanda laboral. Desde esta óptica, la capacitación y orientación docente en el área de evaluación formativa, puede pensarse como uno de los instrumentos más eficaces para mejorar la calidad de la educación en el nivel universitario (Black \& Wiliam, 1998, 2009; NRC, 2000, 2011).

\section{Antecedentes.}

En Europa, según la Estrategia Europa 2020 enmarcada en el EEES (Espacio Europeo de Educación Superior), se ha considerado que todos sus países miembros deban promover en la educación superior de entornos de aprendizaje más interactivos, centrados en el estudiante, con uso eficiente de las Tecnologías de la Información y de la Comunicación (TIC), donde se apueste por la innovación, como parte de la modernización de la educación superior (Gámiz \& Gallego, 2016). Al respecto, es el desarrollo de la metodología combinada, del blended learning, el que ha tenido resultados favorables al considerar la pedagogía con la tecnología, donde se pone de manifiesto la presencialidad y virtualidad como una unidad.

$\mathrm{Al}$ analizar algunas propuestas teóricas encontramos la valoración creciente de modelos centrados en el aprendizaje, donde toma fuerza la enseñanza innovadora (trabajo cooperativo, aprendizaje basado en problemas, desarrollo de proyectos, etc.), donde los estudiantes asumen su rol de actores de su propio proceso de aprendizaje y siendo el profesor el mediador o articulador de los entornos y experiencias de dichos aprendizajes (Gargallo, Jiménez, Martínez, Giménez y Pérez, 2017). Es de esta manera, según el presente enfoque, importante no sólo el método utilizado sino también el entorno de aprendizaje de los estudiantes.

En un estudio realizado sobre los métodos centrados en el aprendizaje, que tuvo como hipótesis que la aplicación de dichos métodos comportará diferencias estadísticamente significativas, obtuvo como uno de sus resultados que existe una mejora de las estrategias de aprendizaje en los estudiantes y que dicha mejora es mayor en las estrategias de carácter motivacional, metacognitivo, de búsqueda y selección y de procesamiento y uso, donde la dinámica docente y evaluativa implementada se presenta con mayor influencia positiva (Gargallo, Garfella, Sahuquillo, Verde \& Jiménez, 2015).

En las estrategias de aprendizaje (escalas de: adquisición, codificación, recuperación y de apoyo), en cuanto a hombres y mujeres, el género sí influye en la elección de dichas estrategias, siendo la más influyente en los hombres y, por otro lado, la preferencia total de las mujeres estuvo inclinada hacia la escala de codificación y apoyo, obteniendo valoraciones más altas. Asimismo, en la escala de adquisición las estrategias más importantes estuvieron en: el subrayado lineal, luego la exploración teórica el repaso mental. En cuanto a la escala de codificación: las nemotecnias, la elaboración de imágenes, las autopreguntas y el parafraseado, constituyeron las estrategias de aprendizaje más importantes tanto para hombre y mujeres. En el mismo sentido, destacó la escala de recuperación, donde la planificación de respuestas también fue la más importante. Y, finalmente, en la escala de apoyo, resaltaron: el automanejo, autoconocimiento, la autoinstrucción y el autocontrol, seguido de la motivación intrínseca y extrínseca (Acevedo, Durán \& Alvis, 2015).

Al analizar las estrategias de aprendizaje, según Acevedo (2016), las mujeres mostraron una mayor fortaleza en el uso de estrategias metacognitivas (planificar y revisar sus procesos) y motivacionales, y en cuanto a los varones su mayor uso fue las estrategias cognitivas (memorizar, organizar y elaborar información). Asimismo, es oportuno señalar que las estrategias que mayor 
uso hacen los estudiantes de mayor rendimiento académico fueron las metacognitivas (la planificación: control previo del estudio, y la revisión: puesta en marcha del proceso al finalizar al finalizar). En suma, es comprender el antes (planificación de la meta y los recursos), durante (supervisión y monitorización del proceso) y después (logros alcanzados).

\section{Método}

La metodología se basó en la aplicación del enfoque cualitativo con un diseño de estudio de caso en el que se consideró la caracterización del empleo de las estrategias de aprendizaje por parte de un grupo de estudiantes de educación superior. A través de la observación directa y la entrevista persona a persona, se identificaron aquellas estrategias que marcaban la praxis constante de los estudiantes y las conversaciones realizadas con 13 de ellos, que tuvieron como propósito el profundizar en los hallazgos venidos de la observación con el fin de interpretar la acción de éstos. El rigor científico de la investigación estuvo demarcado por los criterios de credibilidad y confirmabilidad expresados en la selección de los sujetos participantes y el diálogo auténtico plasmado en las entrevistas realizadas.

\section{Resultados}

A partir de las observaciones realizadas se identificaron las estrategias de aprendizaje más recurrentes por parte de los estudiantes. Ello fue acompañado por la interpretación producto de las entrevistas.

\section{Tabla 1.}

Estrategias metacognitivas

\section{Empleo de la metacognición}

Cuando estudio intento realizar primero aquellas actividades las cuales me resultan más fáciles de manejar...lo difícil lo dejo para después (entrevistado $5)$.

Intento mejorar aquello que me resulto erróneo antes... (entrevistado 2).

Me es difícil establecer un horario el cual me permita estudiar en casa ... el trabajo y otras ocupaciones me lo impiden (entrevistado 1).

Con respecto a las técnicas... confío en la representación gráfica de las ideas... creo que es la forma más práctica de estudiar para un examen (entrevistado 11).

Estimo que el resultado de mis evaluaciones es bueno ya que me preparo para ello (entrevistado 7). 
Tabla 2.

Procesamiento de la información.

Acerca del empleo de estrategias de procesamiento

Procuro emplear recursos que antes me sirvieron para poder enfrentar nuevas tareas (entrevistado 10).

Al estudiar, el hecho de contextualizar mis acciones de la vida diaria me ayuda muchas veces a resolver problemas propuestos en las asignaturas (entrevistado 9).

Tengo la capacidad de asociar lo que aprendo en un curso para aplicarlo en otros cursos diferentes (entrevistado 10).

El trabajar de manera gráfica como por ejemplo haciendo dibujos, tablas.... me ayudan mucho al momento de estudiar (entrevistado 8).

El trabajo particular que realizo me dificulta trabajar con resúmenes o escritos de ayuda al momento de estudiar (entrevistado 13).

Una forma de ver panorámicamente el tema a estudiar es a través de los subtítulos sugeridos por el profesor (entrevistado 4).

Me resulta difícil comprender algunos documentos que el docente me facilitan ... la terminología se convierte en una enorme dificultad (entrevistado 5). Nuestro docente es un buen profesor pero quizá el no ser del área especializada dificulta que nos pueda sacar de más de una duda (entrevistado 11).

\section{Tabla 3.}

Acerca del contexto.

Acerca de estrategias dirigidas al control del contexto
Si no trabajara particularmente, lograría mejores resultados académicos (entrevistado 6).

Aquellos resultados alentadores me hacen suponer que puedo hacer las cosas mucho mejor (entrevistado 9).

Aquel docente reconoce mi esfuerzo y eso me motiva a seguir y continuar seguir mejorando (entrevistado 11)

Algunos de mis compañeros me dicen que tienen en casa un espacio físico exclusivo para el estudio.... En mi caso, no ocurre eso... creo que me ayudaría para tener mis cosas en orden (entrevistado 9).

Cuando trabajamos en grupo siento que puedo rendir académicamente más (entrevistado 2 ). 


\section{Discusión}

A partir de los hallazgos asumimos que, si bien es cierto el marco teórico referencial nos conduce a la existencia de toda una gama de estrategias de aprendizaje, en este caso, estudiado cualitativamente, las estrategias coexistentes se resumen en estrategias metacognitivas, de procesamiento de la información y las estrategias de contexto. De esta manera, podemos asumir que las experiencias exitosas de los estudiantes acerca de cómo superar las dificultades académicas de aprendizaje se constituyen en referentes para cómo actuar de ahí en adelante. El asumir una acción a partir del éxito logrado se convierte en un modus operandi.

Otro hallazgo importante es la inclinación hacia el aprendizaje a través de la expresión gráfica. Al parecer, el contexto y las particularidades propias del grupo de estudiantes, estudiantes y trabajadores a la vez motivan a que, primero, resulte difícil el tener tiempos pre establecidos para estudiar, y, por otro lado, a que las estrategias de aprendizaje se apliquen de manera muy breve, sin considerar largos espacios de tiempo de lectura, ello conlleva a que el interés por la síntesis de la información plasmada en un gráfico centre el interés del estudiante. Se resalta aquí el deseo de que sería otro panorama el hecho de que se disponga de más tiempo para dedicarlo al estudio. El hecho de no contar con un espacio físico exclusivo en casa para estudiar es identificado como un obstáculo, pero no de manera grave o pronunciada. Quizá también un punto a resaltar estaría vinculado con la experiencia misma del estudiante, quien aprovecha los recursos o acciones que le fueron de utilidad antes y que emplea en los casos sucesivos no porque estratégicamente sea éste el procedimiento más adecuado, sino por una cuestión de efectividad. También sería importante el comentar que la experiencia del día a día extra académicamente es aprovechada por los alumnos para vincular lo aprendido con los nuevos temas o tareas por enfrentar.

Una constante al momento de la charla con los estudiantes es la predisposición al estudio a través del vuelo de pájaro (revisión rápida de títulos, subtítulos, imágenes, etc.) plasmados en los textos. Sucede que los tiempos disponibles por los estudiantes son cortos y ello aleja, en gran parte de ellos, la posibilidad de la lectura paciente, calmada y profunda. En muy pocos casos se observa una negativa hacia la capacidad del docente, solamente en casos particulares se observa la dificultad temática observada en los profesores. Se reconoce el empleo en aula de los trabajos colaborativos como una motivación más para poder aprender. Si se resalta también el aspecto motivador de los profesores frente al logro alcanzado por los estudiantes lo cual es muy valorado.

A partir de esta intervención cualitativa, se pudieron observar una serie de particularidades con relación con las estrategias de aprendizaje más recurrentes en un grupo de estudiantes de educación superior. La observación directa y las entrevistas persona a persona ayudaron a la comprensión acerca de la temática abordada.

Se considera, en principio, el empleo de estrategias metacognitivas las cuales se traducen en lo que hizo el estudiante alguna vez y le resultó efectivo, ello se convierte en un recurso constante que se aplica a la mayor cantidad de circunstancias posibles (por una cuestión de eficacia casuística).

El otro aspecto a resaltar es el procesamiento de la información, lo que se plasma, entre otras cosas, en la vinculación de lo que se conoce como parte de la experiencia con las diferentes tareas académicas. Otro punto relevante es la predilección por la información gráfica, lo cual es una forma abreviada de poder asimilar los contenidos propuestos en las asignaturas. 
Finalmente, el contexto se convierte, a partir de lo observado y dialogado, en una característica coexistente en este grupo humano. El hecho de estudiar y trabajar, la motivación docente ante el avance de los logros estudiantiles, las técnicas o dinámicas empleadas por el docente son rescatadas por los estudiantes y entendidas como impulsores positivos al momento de aprender.

En definitiva, se ha tratado en esta aproximación cualitativa al objeto de estudio sobre las estrategias de aprendizaje de estudiantes en el ámbito universitario de dibujar un patrón de metodología de aprendizaje variado, autónomo y dirigido. Es una línea que, si bien se ha desarrollado en otros contextos, ha resultado productiva en el contexto realizado, de cara a mejorar las estrategias de aprendizaje cambiando las estrategias y recursos de enseñanza, por un lado, y las modalidades y naturaleza de evaluación, por otra. Propósitos todos ellos loables, que aconsejan seguir escudriñando el tópico de esta investigación y perpetuar los esfuerzos emprendidos para conseguir avances epistemológicos en el corpus teórico, y que se traduzcan en mejoras reales de la praxis acto didáctico universitario.

\section{Referencias}

Acevedo, D., Durán, M., \& Alvis, A. (2015). Identificación de estrategias de aprendizaje de estudiantes de ingeniería de alimentos en los cursos balance de materia, transporte de fluidos y operaciones unitarias. Formación universitaria, 8(6), 31-38. Doi: https://doi.org/10.4067/S0718-50062015000600005

Acevedo, M. (2016). Estrategias de aprendizaje, con relación al rendimiento académico y tiempo en alcanzar el grado universitario en enfermería (Tesis doctoral). Universidad de Málaga, España. Recuperado de: https://bit.ly/2D0WAsV

Aznar, P., Ull, M. A., Piñero, A., \& Martínez-Agut, M. P. (2013). La sostenibilidad en la formación universitaria: Desafíos y oportunidades. Educación XX1, 17(1), 133-158. Doi: https://doi.org/10.5944/educxx1.17.1.10708

Bahamón, M., Vianchá, M., Alarcón, L., \& Bohórquez, C. (2012). Estilos y estrategias de aprendizaje: una revisión empírica y conceptual de los últimos diez años. Pensamiento Psicológico, (1), 129-144. https://doi.org/10.11144/183

Black, P. \& Wiliam, D. (2009). Developing the theory of formative assessment. Educational Assessment, Evaluation and Accountability, 21, 5-31.

Black, P. \& Wiliam, D. (1998). Assessmentand classroom learning. Assessment in Education, 5(1), 7-74. Doi: https://doi.org/10.1007/s11092-008-9068-5

Blázquez, J. J., Chamizo, J., Cano, E. I., \& Gutiérrez, S. (2013). Calidad de vida universitaria: Identificación de los principales indicadores de satisfacción estudiantil. Revista de Educación, (362), 458-484. Recuperado de: https://recyt.fecyt.es/index.php/Redu/article/view/14343

Buscà, F., Pintor, P., Martínez, L., \& Peire, T. (2010). Sistemas y procedimientos de Evaluación Formativa en docencia universitaria: Resultados de 34 casos aplicados durante el curso académico 2007-2008. Estudios Sobre Educacion, 18(18), 255-276. Recuperado de: https://dadun.unav.edu/bitstream/10171/9829/2/ESE_18_11.pdf

Cabero, J., \& Marín, V. (2013). Percepciones de los estudiantes universitarios latinoamericanos sobre las redes sociales y el trabajo en grupo. RUSC. Revista de Universidad y Sociedad Del Conocimiento, 10(2), 219-235. Doi: http://doi.dx.org/10.7238/rusc.v10i2.1728

Delors, J. (1996). Los cuatro pilares de la educación. La Educación Encierra Un Tesoro, XXVIII (Diciembre), 91-103. Doi: https://doi.org/10.1017/CBO9781107415324.004 
Fernández, E., Bernardo, A., Suárez, N., Cerezo, R., Núñez, J. C., \& Rosario, P. (2013). Predicción del uso de estrategias de autorregulación en educación superior. Anales de Psicología, 29(3), 865-875. Doi: https://doi.org/10.6018/analesps.29.3.139341

Gallego Ortega, J.L., \& Rodríguez Fuentes, A. (2013). Evaluación de la competencia comunicativa de estudiantes del Magisterio. REVALUE Revista de Evaluación Educativa, 2(1), 110-132. Recuperado de: http://revalue.mx/revista/index.php/revalue/article/view/56

Gallego Ortega, J.L., \& Rodríguez Fuentes, A. (2015). Communication skills training in trainee primary school teachers in Spain. Journal of Teacher Education for Sustainability, 17(1), 8597. Doi: https://doi.org/10.1515/jtes-2015-0007

Gallego, J.L., \& Rodríguez, A. (2017). Alternancia de roles en la evaluación universitaria: docentes y discentes evaluadores y evaluados. REDU. Revista de Docencia Universitaria, 15(2), 349-366. Doi: https://doi.org/10.4995/redu.2017.8780

Gámiz, V. \& Gallego, M. J. (2016). Modelo de análisis de metodologías didácticas semipresenciales en Educación Superior. Educación XX1, 19(1), 39-61. doi:10.5944/ educXX1.13946

García, A., \& Tejedor, F. J. (2017). Percepción de los estudiantes sobre el valor de las TIC en sus estrategias de aprendizaje y su relación con el rendimiento. Educacion XX1, 20(2), 137-159. Doi: https://doi.org/10.5944/educXX1.13447

Gargallo, B., Garfella, P., Sahuquillo, P., Verde, I., \& Jiménez, M. (2015). Métodos centrados en el aprendizaje, estrategias y enfoques de aprendizaje en estudiantes universitarios. Revista de Educación, (370), 229 - 281. Doi: https://doi.org/10.4438/1988-592X-RE-2015-370-304

Gargallo, B., Jiménez, M., Martínez, N., Giménez, J., \& Pérez, C. (2017). Métodos centrados en el aprendizaje, implicación del alumno y percepción del contexto de aprendizaje en estudiantes universitarios. Educación XX1, 20(2), 161-187. Doi: https://doi.org/10.5944/educxx1.15.1.153

Hamodi, C., Pastor, V. M. L., \& Pastor, A. T. L. (2014). Medios, técnicas e instrumentos de evaluación formativa y compartida del aprendizaje en educación superior. Perfiles Educativos, 37(147), 146-161. https://doi.org/10.1016/j.pe.2015.10.004

Herrera, L., \& Lorenzo, O. (2009). Estrategias de aprendizaje en estudiantes universitarios. Un aporte a la construcción del Espacio Europeo de Educación Superior. Educación y Educadores, 12(3), 75-98. Recuperado de: http://educacionyeducadores.unisabana.edu.co/index.php/eye/article/viewFile/1532/1838

Larrán, J. M., \& Andrades, F. J. (2015). Análisis de la responsabilidad social universitaria desde diferentes enfoques teóricos. Revista Iberoamericana de Educacion Superior, 6(15), 91107. https://doi.org/10.1016/S2007-2872(15)30005-6

National Research Council (NRC) (2000). Knowing what students know:The science and design of educational assessment. Washington DC:National Academy Press.

National Research Council (NRC) (2011). A framework for K-12 science Education: Practices, crosscutting concepts, and core Ideas. Committee on a Conceptual Framework for New $K$ 12 Science Education Standards. Washington, DC: The National Academies Press.

Pintrich, P. \& García, T. (1993). Intraindividual differences in students' motivation and self regulated learning. German Journal of Educational Psychology, 7(3), 99-107. Recuperado de: http://psycnet.apa.org/record/1994-19471-001

Porto, A. M., Brenlla, J., Barca, A., Almeida, L. S., \& Peralbo, M. (2012). Motivación escolar y rendimiento: impacto de metas académicas, de estrategias de aprendizaje y autoeficacia. Anales de Psicología, 28(3), 848-859. Doi: https://doi.org/10.6018/analesps.28.3.156101

Rizo, F. M. (2013). Dificultades para implementar la evaluación formativa revisión de literatura. Perfiles Educativos, 35(139), 128-150. Doi: https://doi.org/10.1016/S0185- 
2698(13)71813-0

Rodríguez Fuentes, A., Caurcel, M.J. \& Ramos, A.M. (Dirs.) (2008). Didáctica en el EEES: Guías de Trabajo Autónomo. Madrid: Editorial EOS.

Rodríguez, A. \& Rodríguez, L. (2016). Algoritmo de evaluación del potencial educativo de los espacios arquitectónicos en centros de Formación Profesional. Revista de Educación de la Universidad de Granada REUGRA, 23, 133-150. Recuperado de: http://reugra.es/index.php/reugra/article/view/42

Rodríguez, A., Ayllón, B., Gallego, J. L., \& Gómez, I. A. (2017). The Communication Skills of Future Teachers during their Initial Training. Multidisciplinary Journal of Educational Research RMIE, 7(1), 88-118. Doi: https://doi.org/10.17583/remie.2017.2200

Rodríguez, L. Rodríguez, A., \& Gallego, J.L. (2015). Valoración de los espacios arquitectónicoeducativos de la Formación Profesional en España. REVALUE Revista de Evaluación Educativa, 4(1). Recuperado de: http://revalue.mx/revista/index.php/revalue/issue/view/9

UNESCO (1998). La educación superior en el siglo XXI. Visión y acción. Conferencia Mundial sobre la Educación Superior. ED-98/CONF.202/5, 1-74. Recuperado de: http://unesdoc.unesco.org/images/0011/001136/113602So.pdf

Vallés, C., Ureña, N., \& Ruiz, E. (2011). La Evaluación Formativa en Docencia Universitaria. Resultados globales de 42 estudios de caso. Revista de Docencia Universitaria, 9(1), 135158. Recuperado de: http://red-u.net/redu/files/journals/1/articles/197/public/197-203-2PB.pdf 